Dayani Galato

Jaqueline Madalena ${ }^{2}$

Greicy Borges Pereira ${ }^{2}$

\section{Automedicação em estudantes universitários: $a$ influência da área de formação}

\author{
Self-medication among university students: \\ the influence of the field of study
}

Programa de Mestrado em Ciências da Saúde, Núcleo de Pesquisa em Atenção Farmacêutica e Estudos de Utilização de Medicamentos (NAFEUM), Universidade do Sul de Santa Catarina (UNISUL). Avenida José Acácio Moreira 787, Dehon. CEP 88704-900 Tubarão SC.dayani.galato@unisul.br ${ }^{2}$ Curso de Farmácia, Universidade do Sul de Santa Catarina.

\begin{abstract}
The scope of this paper was to investigate the influence of a university student's field of study upon self-medication. A cross-sectional study was conducted through the administration of questionnaires on the profile of the respondents, self-medication and other healthcare-related issues. In all, 342 students from different fields of study were interviewed, 81 of which were from the health area. Of the respondents, 37\% reported self-medicating in the last fortnight. The most common health problems for self-medication in this period were general pain (90.4\%) and analgesics and antipyretics were the most common form of medication. It was observed that being female ( $p=0.049)$, as well as the fact of having health insurance $(p=0.036)$, were significantly associated with self-medication and that studying in the health area was not associated with self-medication (0.139). However, it was found that the influence of advertising $(p<0.001)$, old prescriptions ( $p=0.041$ ), pharmacists or pharmacy employees ( $p=0.005)$, as well as friends, neighbors and relatives $(p=0.003)$ were more significant among university students who were not within the health area, though the influence of acquired knowledge $(p<0.001)$ is more significant among students in the health area.
\end{abstract}

Key words Self-medication, Students, Higher education, Brazil
Resumo Investigar a influência da área de formação de universitários na prática da automedicação. Estudo transversal com aplicação de questionários sobre o perfil dos entrevistados, a prática da automedicação e outras questões relacionadas ao manejo da saúde. Foram entrevistados 342 universitários das diferentes áreas de formação, sendo 81 da área da saúde. Destes, 37,0\% referiram ter se automedicado nos últimos quinze dias. Neste período os problemas de saúde mais comuns para esta prática foram a dor em geral $(90,4 \%)$ adotando-se principalmente analgésicos e antitérmicos. Observou-se que ser mulher $(p=0,049)$ e possuir plano de saúde $(p=0,036)$ associaramse significativamente a automedicação e que ser da área de saúde não está associado à prevalência desta prática $(p=0,139)$. Contudo, identificouse que a influência da propaganda $(p<0,001)$, de prescrições antigas ( $p=0,041)$, de farmacêuticos ou funcionários da farmácia $(p=0,005)$ e de amigos, vizinhos e familiares $(p=0,003)$ são significativas em universitários que não são da área de saúde e que a influência do conhecimento próprio $(p<0,001)$ é significativo em estudantes da área da saúde.

Palavras-chave Automedicação, Estudantes, Educação superior, Brasil 


\section{Introdução}

A automedicação é a prática de utilizar medicamentos sem prescrição ${ }^{1}$, estando inclusa dentro do conceito de autocuidado ${ }^{2}$. Neste caso, relacionado com o cuidado que as pessoas têm de prevenir as doença e manter a saúde abrangendo a higiene (geral e individual); a alimentação (tipo e qualidade dos alimentos ingeridos); estilo de vida (atividades esportivas, lazer, etc.); os fatores ambientais (condições de vida, hábitos sociais, etc.); os fatores socioeconômicos (renda, crenças culturais, etc.); e a automedicação².

A automedicação responsável é definida como a prática pela qual indivíduos tratam seus problemas de saúde com medicamentos aprovados e disponíveis para serem adquiridos sem prescrição, e que sejam seguros e efetivos quando utilizados como indicado ${ }^{2}$.

Vários trabalhos ${ }^{3-6}$ têm demonstrado que esta prática é muito comum, em idosos pode chegar a $80 \%^{7}$ e na população em geral a $46 \%{ }^{8}$ quando investigado os últimos 90 dias. Em trabalhos realizados com estudantes estes valores foram superiores a $70 \%{ }^{3,5}$.

Mesmo que muitas vezes esta prática possa ter resultados favoráveis (melhora de sintomas ou resolução do problema de saúde), outras vezes pode trazer prejuízos à saúde do indivíduo (mascaramento dos problemas de saúde, intoxicação, reações adversas, interações medicamentosas, desenvolvimento de resistência entre outros) $)^{9,10}$.

Segundo Tomasi et al. ${ }^{11}$ o risco dessa prática está correlacionado com o grau de instrução e informação dos usuários sobre medicamentos, bem como com a acessibilidade dos mesmos ao sistema de saúde. Neste contexto, buscando investigar a influência da área de formação da graduação na prática da automedicação foi realizado um estudo com universitários.

\section{Métodos}

\section{Delineamento do estudo e local de realização}

Este estudo possui um delineamento transversal utilizando a técnica de entrevista.

A população estudada envolveu universitários de uma instituição de ensino superior do Sul do Estado de Santa Catarina, localizada a 130 quilômetros da Capital do Estado, Florianópolis. Este campus da Universidade possui atual- mente 42 cursos que estão organizados em quatro áreas: gestão e jurídica (Sociais Aplicadas segundo o CNPq), tecnológica (Ciências Exatas e da Terra, Engenharias e Ciências Agrárias segundo o CNPq), saúde (Ciências da Saúde, Ciências Biológicas e psicologia das Ciências humanas segundo o CNPq), comunicação e educação (Linguísticas, letras e artes segundo o $\mathrm{CNPq}$ ).

\section{Procedimento amostral, critérios de inclusão e de exclusão}

A amostra mínima desta pesquisa foi calculada levando-se em consideração um erro de 5\%, uma prevalência estimada de automedicação nos últimos 15 dias de 50\% (o que maximiza a amostra), um intervalo de confiança de $95 \%$, levando em consideração uma população de 3726 estudantes matriculados naquele período, totalizando um mínimo de 349 sujeitos.

Adotou-se um processo de amostragem em duas etapas. Na primeira foi realizado o sorteio de 12 cursos, sendo três representantes de cada uma das áreas apresentadas anteriormente.

Posteriormente, ocorreu por conveniência a seleção das turmas de alunos dos cursos sorteados para as entrevistas. Como critério de inclusão para os entrevistados foi adotado o fato de pertencerem a turmas que tivessem cursado pelo menos 50\% dos respectivos cursos, pois assim já teriam o conhecimento da área de formação. Para esta seleção, os proponentes solicitaram a permissão de realização da pesquisa aos coordenadores de curso que auxiliaram na definição das turmas para compor a amostra e dos melhores horários para a realização da coleta de dados, neste caso a conveniência foi a facilidade de acesso as turmas. Foram entrevistados no mínimo 30 alunos por curso.

Como critérios de exclusão foi o de ter cursado anteriormente outra faculdade e não assinar o termo de consentimento livre e esclarecido.

\section{Instrumento e variáveis da pesquisa}

O Instrumento de coleta de dados composto por questões fechadas está estruturado em duas partes, na primeira é descrito o perfil dos entrevistados como: curso, idade, sexo e classificação econômica. Na segunda foram descritas questões relacionadas à automedicação (sobre essa prática em algum momento da vida e; nos últimos 15 dias; fatores que influenciam; medicamentos utilizados e; problemas de saúde em que esta prática é adotada) e sobre outras questões 
relacionadas ao manejo da saúde (frequência de procura a médicos e farmacêuticos no último ano; leitura de bula; presença de plano de saúde).

Antes da aplicação do instrumento para a coleta de dados ele foi testado em estudantes universitários a fim de avaliar a legibilidade do mesmo, para isto foi escolhida uma turma do curso de Farmácia, sendo estes dados inclusos na pesquisa, pois não houve alteração no questionário nesta etapa.

É importante salientar que neste trabalho foram definidos os últimos 15 dias ${ }^{12,13}$ como período de recordatório para a automedicação.

\section{Métodos estatísticos e análise de dados}

Os resultados foram apresentados adotando-se para variáveis categóricas números absolutos e proporções e para variáveis numéricas medidas de tendência central e de dispersão e de amplitude de variação.

Foram realizadas também as análises associação utilizando o teste do qui-quadrado ou a prova exata de Fischer para avaliar a influência do perfil dos universitários na prática da automedicação, sendo adotado como significância $\mathrm{p}<0,05$. Também foi estimada a razão de prevalência (IC 95\%) na análise das variáveis categóricas.

Adotou-se o programa Estatístico SPSS 19.0 para a realização das análises.

Este trabalho foi aprovado pelo Comitê de Ética em Pesquisa da Universidade do Sul de Santa Catarina.

\section{Resultados}

Foram entrevistados 384 estudantes divididos entre os 12 cursos sorteados nas quatro áreas. Destes foram excluídos 5 que não assinaram o termo de consentimento livre e esclarecido, 2 por não terem cursado $50 \%$ do curso e 35 por já terem cursado outra faculdade. Totalizando 342 entrevistados. Os cursos sorteados na área da saúde foram: Farmácia, Fisioterapia e Medicina; na área de Gestão: Contabilidade, Administração e Direito. Na área de Tecnológicas: Química Industrial, Engenharia Química e Tecnologia em Redes de Computadores; e, na área de Comunicação: Pedagogia, Letras Português/Inglês e Tecnologia em Design de Moda.

Do total de entrevistados, $81(23,7 \%)$ eram da área da saúde, 237 (69,7\%) eram do sexo feminino, quanto à idade, a mesma variou de 18 a 49 apresentando a média de 22,9 \pm 4,2 anos.
Em relação à automedicação, 330 (96,5\%) afirmaram que já realizaram essa prática, 137 $(41,9 \%)$ já utilizaram sobras de medicamentos anteriores e $125(37,0 \%)$ utilizaram medicamentos sem prescrição nos últimos 15 dias. De todas as pessoas que afirmaram que se automedicaram em algum momento da vida $21(6,4 \%)$ relataram ter apresentado problemas, sendo que 1 relatou intoxicação, 3 mascaramento de outros problemas de saúde, 2 resistência bacteriana e as demais reações adversas ao medicamento.

Os problemas de saúde cujo manejo foi realizado por automedicação estão apresentados na Tabela 1, juntamente com os medicamentos mais utilizados, os motivos para a prática e as influências para a automedicação.

Observou-se que existe uma associação significativa em realizar a automedicação através de sobras de tratamentos anteriores quando os universitários afirmam sofrer influência de familiares, vizinhos e amigos ( $p=0,005)$ ou de prescrições antigas $(\mathrm{p}<0,001)$.

Tabela 1. Prática da automedicação: problemas de saúde e medicamentos adotados por estudantes de uma universidade do sul do Brasil, 2009.

\begin{tabular}{lr}
\hline \multicolumn{1}{c}{ Variáveis } & n (\%) \\
\hline Problemas de saúde $(\mathrm{n}=125)$ & \\
Dor em geral & $113(90,4)$ \\
Gripes e resfriado & $25(20,0)$ \\
Dor de estômago & $5(4,0)$ \\
Rinite & $5(4,0)$ \\
Tosse & $4(3,2)$ \\
Outros & $26(20,8)$ \\
Medicamentos (n = 216) & \\
Paracetamol & $34(14,3)$ \\
Paracetamol + associação & $14(6,5)$ \\
Dipirona & $26(12,0)$ \\
Dipirona + associação & $19(8,8)$ \\
Orfenadrina + Dipirona & $13(6,0)$ \\
Belladona e derivados & $5(2,3)$ \\
Motivos (n = 343) & \\
Praticidade e comodidade & $204(59,5)$ \\
Facilidade de compra na farmácia & $167(48,7)$ \\
Falta de acesso aos serviços de saúde & $21(6,1)$ \\
Falta de dinheiro & $12(3,5)$ \\
Outros & $18(5,2)$ \\
Influências para a automedicação (n = 356) & \\
Farmacêutico ou funcionários da farmácia & $175(49,2)$ \\
Familiares, vizinhos e amigos & $174(48,9)$ \\
Conhecimento próprio & $157(44,1)$ \\
Prescrições antigas & $90(25,3)$ \\
Propaganda & $30(8,4)$ \\
&
\end{tabular}


Apenas duas pessoas $(1,8 \%)$ que realizaram a automedicação nos últimos 15 dias afirmaram adotar plantas medicinais. Em relação ao uso de anticoncepcional das 161 mulheres que responderam esta questão $38(23,6 \%)$ afirmaram utilizar este medicamento sem a prescrição de um profissional médico.

Não foram identificadas diferenças significativas entre as variáveis de desfecho (automedicação) e as diferentes áreas de formação. Também não foi observada nenhuma associação quando se analisou separadamente os acadêmicos da área de saúde com as demais áreas (dados não apresentados). No entanto, quando comparada a área da saúde com as demais, em relação às influências relatadas para a prática da automedicação, houve uma associação significativa, conforme apresentado na Tabela 2.

A Tabela 3 apresenta os resultados dos testes de associação entre a variável automedicação nos últimos quinze dias e o perfil dos entrevistados e outras questões relacionadas à saúde.

\section{Discussão}

Em relação à automedicação 96,5\% dos entrevistados já realizaram essa prática em algum momento da vida, segundo Penna et al. ${ }^{5}$ em um estudo realizado com universitários no ano de 2004 , este percentual variou de $75,5 \%$ a $92,0 \%$. Já em outras populações este valor também foi superior a $80 \%$.
Loyola et al. ${ }^{8}$ encontraram uma prevalência de automedicação nos últimos 90 dias de $46 \%$, já em nosso trabalho, este percentual nos últimos 15 dias foi de $37 \%$, sendo menor o percentual provavelmente pelo tempo de recordatório utilizado.

Mesmo que a automedicação faça parte do autocuidado $^{2}$ é importante que seja avaliado se a mesma é realizada de forma responsável ${ }^{1}$, isto significa afirmar que deve ser realizada no contexto do uso racional de medicamentos ${ }^{14}$. Neste caso, é necessário que os estudantes utilizem os medicamentos para as suas necessidades clínicas, que devem ser caracterizadas no contexto da automedicação como transtornos menores, ou seja, problemas autolimitados ${ }^{1}$. Além disso, devem ser selecionadas alternativas terapêuticas, seguras e eficazes ${ }^{14}$ que estejam à venda sem obrigatoriedade da prescrição médica ${ }^{1}$ e devem ser utilizadas nas doses, posologias e tempos de tratamento adequados para que se alcance a maior efetividade e segurança. Ainda neste contexto, é importante destacar que a automedicação não deve ser adotada pelos pacientes ditos especiais, neste caso inclui-se as gestantes, as crianças menores de dois anos, os idosos e os pacientes polimedicados ou com morbidades graves ${ }^{1}$.

Quando não ocorre a adoção desta prática seguindo os critérios de uso racional de medicamentos, tanto o paciente quanto a sociedade podem ter prejuízos. Estes podem estar relacionados ao mascaramento de sintomas de problemas sérios, a seleção de bactérias resistentes, a

Tabela 2. Comparação entre as áreas de formação com as influências relatadas para a prática da automedicação em estudantes de uma Universidade do sul do Brasil, 2009.

\begin{tabular}{|c|c|c|c|c|c|}
\hline Influências & Total & Saúde & $p$ & $\mathbf{R P}$ & IC $95 \%$ \\
\hline Farmacêuticos ou funcionários da farmácia & & & 0,005 & & $1,18-2,57$ \\
\hline Não & 151 & $48(31,8)$ & & 1,73 & \\
\hline Sim & 175 & $32(18,3)$ & & 1,00 & \\
\hline Família, amigos e vizinhos & & & 0,003 & & $1,22-2,68$ \\
\hline Não & 152 & $49(32,2)$ & & 1,81 & \\
\hline Sim & 174 & $31(17,8)$ & & 1,00 & \\
\hline Conhecimento próprio & & & $<0,001$ & & $1,31-1,72$ \\
\hline Não & 169 & $17(10,1)$ & & 1,00 & \\
\hline Sim & 157 & $63(40,1)$ & & 1,50 & \\
\hline Propaganda & & & $<0,001$ & & - \\
\hline Não & 296 & $80(27,0)$ & & - & \\
\hline Sim & 30 & $0(0,0)$ & & & \\
\hline Prescrições antigas & & & 0,041 & & $1,00-2,74$ \\
\hline Não & 236 & $65(27,5)$ & & 1,65 & \\
\hline Sim & 90 & $15(16,7)$ & & 1,00 & \\
\hline
\end{tabular}

p-Nível de significância obtido no teste de Qui-quadrado, RP - Razão de Prevalência estimada; IC - Intervalo de Confiança. 
Tabela 3. Resultados dos testes de associação entre automedicação praticada pelos estudantes de uma universidade do sul do Brasil e seu perfil.

\begin{tabular}{|c|c|c|c|c|c|}
\hline Variáveis & Total & Automedicação $\left(^{*}\right)$ & $p$ & $\mathbf{R P}$ & IC (95\%) \\
\hline Área de concentração & & & 0,139 & & $0,90-1,88$ \\
\hline Outras & 258 & $101(39,1)$ & & 1,30 & \\
\hline Saúde & 80 & $24(30,0)$ & & 1,00 & \\
\hline Sexo & & & 0,049 & & $0,99-1,97$ \\
\hline Feminino & 235 & $94(40,0)$ & & 1,39 & \\
\hline Masculino & 101 & $29(28,7)$ & & 1,00 & \\
\hline Idade & & & 0,950 & & $0,75-1,36$ \\
\hline 23 ou mais & 117 & $43(36,8)$ & & 1,01 & \\
\hline Até 22 & 217 & $79(36,4)$ & & 1,00 & \\
\hline Número de moradores na residência & & & 0,905 & & $0,77-1,35$ \\
\hline Até 3 & 159 & $59(37,1)$ & & 1,02 & \\
\hline Mais de 4 & 170 & $62(36,5)$ & & 1,00 & \\
\hline Classe & & & 0,920 & & $0,72-1,43$ \\
\hline A e B & 264 & $98(37,1)$ & & 1,02 & \\
\hline C e D & 74 & $27(36,5)$ & & 1,00 & \\
\hline Plano de saúde & & & 0,036 & & $1,01-1,84$ \\
\hline Sim & 188 & $78(41,5)$ & & 1,36 & \\
\hline Não & 145 & $44(30,3)$ & & 1,00 & \\
\hline Lê a bula & & & 0,668 & & $0,81-1,40$ \\
\hline Sempre que possível & 139 & $55(39,6)$ & & 1,06 & \\
\hline Outros & 188 & $70(37,2)$ & & 1,00 & \\
\hline Ir ao médico nos últimos 12 meses & & & 0,352 & & $0,80-1,85$ \\
\hline $\operatorname{Sim}$ & 283 & $108(38,2)$ & & 1,21 & \\
\hline Não & 54 & $17(31,5)$ & & 1,00 & \\
\hline Ir a farmácia (orientação) & & & 0,245 & & $0,89-1,58$ \\
\hline Sim & 196 & $78(39,8)$ & & 1,18 & \\
\hline Não & 140 & $47(33,6)$ & & 1,00 & \\
\hline
\end{tabular}

(*) automedicação nos últimos 15 dias. $p$ - Nível de significância obtido no teste de Qui-quadrado, RP - Razão de Prevalência estimada; IC - Intervalo de Confiança.

procura a hospitais por problemas de segurança dos medicamentos, entre outros 9 .

Das pessoas que afirmaram que se automedicaram em algum momento da vida, 6,4\% apresentaram algum tipo de problema. Apesar de não ter sido encontrado trabalhos que demonstrem a prevalência deste fato, alguns descrevem os riscos da prática de automedicação $0^{9,10}$.

No presente trabalho foi observado que o problema de saúde mais citado para o manejo com automedicação foi a dor em $90,4 \%$ das situações, englobando dores de cabeça, cólicas, dor em geral, entre outras. Este problema de saúde também é apresentado em outras pesquisas ${ }^{3,5,10,15-}$ ${ }^{18}$ como o mais comum para a prática da automedicação. Quando investigados os medicamentos selecionados para a automedicação nos últimos 15 dias observou-se que entre os 6 mais utilizados 5 possuem analgésicos, que totalizam so- zinhos 52,3\% dos medicamentos utilizados. Estes resultados são coerentes com o principal problema de saúde que motivou a prática da automedicação, que foi a dor, e encontra subsídios em outros trabalhos descritos na literatura ${ }^{3,5,6,8,15-19}$. No entanto, é importante destacar que em algumas situações o manejo da dor tem sido realizado com outros medicamentos, como por exemplo o uso de antiespasmódicos no caso de cólicas e de relaxantes em problemas musculares.

Entre os motivos que influenciaram na automedicação os mais citados foram a praticidade, facilidade de compra e falta de acesso ao serviço de saúde. Sendo este resultado semelhante ao encontrado por Mussolin ${ }^{4}$ em estudo realizado entre universitários de relações públicas e enfermagem. Neste mesmo estudo, Mussolin ${ }^{4}$ observou que os alunos de enfermagem liam mais a bula. Este resultado também se apresenta seme- 
lhante ao nosso trabalho onde, os estudantes da área da saúde lêem mais a bula em relação às outras áreas.

Com relação à utilização de plantas medicinais como uma alternativa para a prática de automedicação nos últimos 15 dias, apenas duas pessoas relataram utilizar plantas. Esse resultado pode estar relacionado com o fato de que os entrevistados eram em sua maioria jovens, já que a utilização de plantas é mais comum na população idosa ${ }^{7}$.

Quando investigado o uso de sobras de medicamentos, ou seja, de medicamentos já armazenados no domicílio oriundos de tratamentos anteriores observou-se que esta prática associase significativamente naqueles indivíduos que afirmam ser influenciados por familiares ou por antigas prescrições. Estes fatos podem ser possivelmente explicados por experiências exitosas com o medicamento indicado seja pelo familiar ou pelo próprio sujeito da pesquisa.

Uma das hipóteses levantadas pelos investigadores para a realização deste trabalho era de que possuir formação na área de saúde poderia influenciar na prevalência da prática da automedicação, no entanto, esta informação não foi confirmada. Por outro lado, mesmo que a prevalência da automedicação não seja alterada significativamente pela área de formação, observou-se que todas as influências investigadas para esta prática diferem significativamente em relação à área de formação, ser a saúde ou outra.

Desta forma, verificou-se que a propaganda de medicamentos, prescrições antigas, o farmacêutico ou o funcionário da farmácia e os familiares vizinhos e amigos, influenciam significativamente os universitários de outras áreas de formação. De outra forma, o conhecimento próprio a respeito do problema de saúde e do medicamento tem influenciado significativamente os universitários da área da saúde. Estes resultados são importantes, pois, podem nortear as estratégicas para a promoção do uso racional de medicamentos dependendo do público que se pretende atuar.

Um dado alarmante deste trabalho foi o fato de que $23,6 \%$ das mulheres que utilizavam anticoncepcional o faziam sem prescrição médica. Segundo a Organização Mundial de Saúde ${ }^{20}$ existem critérios médicos de elegibilidade de anticoncepcionais orais que devem ser respeitados na seleção deste medicamento. Em um estudo publicado por Matarezi et al. ${ }^{21}$, na região de Maringá, no estado do Paraná, um terço das usuárias de anticoncepcionais orais o faziam sem nenhu- ma consulta médica prévia, dado este que fica ainda mais importante quando estes mesmos autores afirmam que praticamente metade dessas mulheres apresentava alguma contra-indicação para o seu uso.

Foi observado que as mulheres se automedicaram nos últimos 15 dias de uma forma mais significativa $(p=0,049)$ que os homens, isto tem sido descrito em outros trabalhos ${ }^{8}$. Este comportamento pode ser explicado pelo fato das mulheres realizarem mais o autocuidado e consequentemente a automedicação.

A idade não teve associação com a automedicação, vários trabalhos têm demonstrado não haver padrões de comportamento entre estas duas variáveis ${ }^{7,8}$.

Diferente do apresentado por Loyola Filho et al. ${ }^{8}$, que investigaram este tema em pacientes adultos, o número de visitas ao médico ou ao farmacêutico nos últimos 12 meses não apresentou associação a automedicação nos últimos 15 dias, assim como o número de moradores no domicílio que também não apresentou associação significativa.

Damasceno et al. $^{3}$ observaram que a renda familiar não influenciou a prática da automedicação, assim como no nosso trabalho que também não apresentou associação significativa quando comparado à classificação socioeconômica com a prática da automedicação nos últimos 15 dias.

Neste trabalho foi observado que pessoas com planos de saúde automedicam-se de forma mais significativa do quem não os possuem, este resultado é controverso daqueles apresentados por Mussolin ${ }^{4}$ em seu trabalho realizado com universitários e por Vilarino ${ }^{22}$ em seu estudo com a população em geral. Uma das hipóteses levantadas para explicar este fenômeno seria o fato de que pessoas que possuem planos de saúde poderiam estar reutilizando mais antigas prescrições, a qual não foi confirmada em nosso trabalho.

Uma limitação deste estudo é o fato de não ter sido analisado se a automedicação realizada pelos entrevistados era adequada ou não levando em consideração o perfil dos sujeitos e as características do problema de saúde, no entanto, justifica-se que este não era objetivo deste estudo.

\section{Conclusões}

Observou-se que a automedicação é bastante comum nesta população, no entanto, não houve diferença entre esta prática em função da área de 
formação. Uma contribuição importante deste trabalho diz respeito às influências que levam à prática da automedicação, ou seja, amigos, vizinhos e familiares, da propaganda, de farmacêuticos ou funcionários da farmácia e de prescrições antigas influenciam significativamente estudantes de outras áreas, enquanto o conhecimento próprio influencia mais estudantes da área da saúde. Estes dados podem, portanto, ser utilizados na definição de estratégias voltadas a promoção do uso racional de medicamentos.

\section{Colaboradores}

D Galato, J Madalena e GB Pereira participaram igualmente de todas as etapas de elaboração do artigo. 


\section{Referências}

1. The International Pharmaceutical Federation, The World Self-Medication Industry. Responsible SelfMedication [Internet document] 1999. [cited 2010 Feb 23]; [3p]. Available from: http://www.wsmi.org/ pdf/fip.pdf

2. World Health Organization. The role of the pharmacist in self-care and self-medication. The Hangue: World Health Organization [Internet document] 1998 [cited 2010 Feb 22]; [21 p.]. Available from: http://www.opas.org.br/medicamentos/site/UploadArq/who-dap-98-13.pdf

3. Damasceno DD, Terra FS, Zanetti HHV, D'Andréa ED, Silva HLR, Leite JA. Automedicação entre graduandos de enfermagem, farmácia e odontologia da Universidade Federal de Alfenas. Reme Rev Min Enferm 2007; 11(1):48-52.

4. Mussolin NM. A automedicação: um estudo entre universitários de enfermagem e de relações públicas [dissertação]. São Paulo (SP): Secretaria da Saúde. Coordenação dos Institutos de Pesquisa. Programa de Pós-Graduação em Ciências; 2004.

5. Penna AB, Borges CC, Batista RD, Siqueira IMC. Análise da Prática da Automedicação em Universitários do Campus Magnus - Unipac - Barbacena, MG. In: Anais do $2^{\circ}$ Congresso Brasileiro de Extensão Universitária [anais na internet] 2004 Set 12-15; Belo Horizonte, Brasil [acessado 2011 jul 18]. Disponíve em: http://www.ufmg.br/congrext/Saude/Saude20. pdf

6. Narloch L, Peter APC, Feyer ISS, Ledoux T, Goller DP, Marcelino MA, Martins F. A Automedicação entre os acadêmicos da UFSC. In: $4^{a}$ Semana de Ensino, Pesquisa e Extensão [anais na internet] 2004 Set 22-25; Florianópolis, Brasil [acessado 2011 jul 18]. Disponível em: http://www.sepex.ufsc.br/ anais_4/trabalhos/8.html

7. Cascaes AE, Falchetti ML, Galato D. Perfil da automedicação em idosos participantes de grupos da terceira idade de uma cidade do sul do Brasil. Arq Cat Med 2008; 37(1):63-39.

8. Loyola Filho AI, Uchoa E, Gerra HL, Firmo JOA, Lima-Costa MF. Prevalência e fatores associados à automedicação: resultados do projeto Bambuí. Rev Saude Publica 2002; 36(1):55-62.

9. Associação Médica Brasileira. Automedicação. Rev Assoc Med Bras 2001; 47(4):269-270.

10. Ogawa AI, Kurachi G, Hata HT, Abreu KRS, Lourenço L, Santos LL, Matsumura M, Specian MR, Elias TV, Ivama AM, Melchior SC, Soares DA, Turini B. Estudo comparativo sobre automedicação em estudantes do segundo ano de enfermagem e medicina e moradores do bairro Vila Nova. Espaço Saude [periódico na internet]. 2001 [acessado 2011 jul 18]; 3(2):[13 p]. Disponível em: http://www.ccs.uel.br/espaco parasaude/v3n2/doc/automedicacao.doc

11. Tomasi E, Sant'Anna GC, Oppelt AM, Petrini RM Pereira IV, Sassi BT. Condições de trabalho e automedicação em profissionais da rede básica de saúde da zona urbana de Pelotas, RS. Rev Bras Epidemiol 2007; 10(1):66-74
12. Cabrita J, Ferreira H, Iglesias P, Baptista T, Rocha E, Silva AL, Miguel JP. Estudo do padrão de consumo de medicamentos pelos estudantes da Universidade de Lisboa. Rev Port Sau Pub 2001; 19(2):39-47.

13. Pereira FSVT, Bucaretchi F, Stephan C, Cordeiro R. Automedicação em crianças e adolescentes. J Pediatr 2007; 83(5):453-458.

14. Organización Mundial de la Salud. Promoción del uso racional de medicamentos: componentes centrales. Genebra: Organización Mundial de la Salud [internet document] 2002 [cited 2011 Jul 5]; 5[6 p]. Available from: http://www.who.int/medicinedocs/ collect/edmweb/pdf/s4874s/s4874s.pdf

15. Sá MB, Barros JAC de, Sá MPBO. Automedicação em idosos na cidade de Salgueiro-PE. Rev Bras Epidemiol 2007; 10(1):75-85.

16. Servidoni AB, Coelho L, Navarro ML, Ávila FG, Mezzalira R. Perfil da automedicação nos pacientes otorrinolaringológicos. Rev Bras Otorrinolaringol 2006; 72(1):83-88.

17. Vitor RS, Lopes CP, Menezes HS, Kerkhoff CE. Padrão de consumo de medicamentos sem prescrição médica na cidade de Porto Alegre, RS. Cien Saude Colet 2008; 13(Sup):737-743.

18. Mendoza-Sassi R, Béria JU, Fiori N, Bortolotto A. Prevalência de sinais e sintomas, fatores sociode mográficos associados e atitude frente aos sintomas em um centro urbano no Sul do Brasil. Rev Panam Salud Publica 2006; 20(1):22-28.

19. Bortolon PC, Medeiros EFF, Naves JOS, Karnikowski MGO, Nóbrega OT. Análise do perfil de automedicação em mulheres idosas brasileiras. Cien Saude Colet 2008; 13(4):1219-1226.

20. World Health Organization. Medical eligibility criteria for contraceptive use. $3^{\text {rd }}$ Edition. Geneva: World Health Organization [internet document].2004 [cited 2011 Jul 19]; [176 p]. Available from: http:// whqlibdoc.who.int/publications/2004/9241562668. pdf

21. Matarezi de Souza JM, Pelloso SM, Uchimura NS, de Souza F. Utilização de métodos contraceptivos entre as usuárias da rede publica de saúde do município de Maringá-PR. Rev Bras Ginecol Obstet 2006; 28(5):285-291.

22. Vilarino JF, Soares IC, Silveira CM, Rodel APP, Bortoli R, Lemos RR. Perfil da automedicação em município do sul do Brasil. Rev Saude Publica 1998; 32(1):43-49.

Artigo apresentado em 23/03/2011

Aprovado em 19/07/2011

Versão final apresentada em 21/03/2012 